\title{
Iatrogenic Cushing's Syndrome Due to Pharmacokinetic Interaction of Intra-Ocular Corticosteroids and Lopinavir/Ritonavir in an HIV-Infected Adolescent: A Case Report
}

\author{
Diana F Clarke*, Carole Moloney and Ellen R Cooper \\ Boston Medical Center, Section of Pediatric Infectious Diseases, Boston, USA
}

\begin{abstract}
We report a perinatally HIV infected adolescent on a protease-inhibitor containing antiretroviral regimen who developed iatrogenic Cushing's syndrome while receiving intensive ophthalmic corticosteroid therapy for granulomatous panuveitis. Although pharmacokinetic drug interactions between protease inhibitors and oral/inhaled corticosteroids have previously been described, this is the first report involving ophthalmic administration of corticosteroids and lopinavir/ritonavir in an adolescent.
\end{abstract}

Keywords: Iatrogenic cushing's syndrome; Lopinavir rionavir; Intraocular corticosteroids; Drug interactions

\section{Introduction}

There are many drug interactions between antiretroviral agents and other medications, often complicating the management of HIVinfected patients with co-morbidities. Ritonavir is a potent cytochrome p450 CYP3A4 inhibitor and is frequently used as part of protease inhibitor-based antiretroviral therapy. While it has been recognized that there is the potential for iatrogenic Cushing's syndrome related to co-administration of inhaled and intranasal corticosteroids with protease inhibitors, the potential risk with corticosteroid injections and topical administration has been less well documented. We report a case of an HIV-infected adolescent female who developed iatrogenic Cushing's syndrome and adrenal insufficiency due to pharmacokinetic interaction of intra-ocular corticosteroids and lopinavir-ritonavir

\section{Case Report}

In February 2007, a 14 year old perinatally HIV infected female from Uganda began care in the pediatric infectious disease clinic at Boston Medical Center. An antiretroviral regimen consisting of nevirapine, stavudine, and lamivudine had been initiated at a Ugandan clinic shortly before her arrival in the USA. Initial evaluation on arrival revealed multiple congenital anomalies including solitary kidney, atrial septal defect of the heart, rib anomalies, macrocephaly, developmental delay and hyper-extensible joints. Early in 2008, she was found to have hematuria, proteinuria and eosinophiluria on routine urinalysis. In April of 2008, because of virologic and immunologic failure and concern that her renal involvement was possibly related to drug toxicity or her HIV disease, her antiretroviral regimen was changed to lopinavir/ ritonavir (Kaletra ${ }^{\oplus}$ ) and $\operatorname{Trizivir}^{\oplus}$ (abacavir+zidovudine+lamivudine) all given twice daily at the standard adult doses.

In June of 2008, the patient reported visual changes and was noted to have an abnormal screening ophthalmologic exam. The patient was referred to ophthalmology and a diagnosis of non-infectious unilateral granulomatous panuveitis (left eye) was made. From July $18^{\text {th }}$ until September $3^{\text {rd }}$, intensive ophthalmic corticosteroid regimen was administered which included prednisolone ophthalmic suspension $1 \%$ drops (PredForte $1 \%$ ) and tobramycin $0.3 \%$ +dexamethasone $0.1 \%$ ointment $\left(\right.$ Tobradex $\left.^{\circledast}\right)$. Dosages for topical prednisolone ranged from one drop four times daily to one drop every hour while awake and the dexamethasone ointment was given once daily to four times daily.
Rapid worsening and visual loss were noted over several weeks and the patient received a posterior sub-tenon injection of triamcinolone acetonide $\left(\right.$ Kenalog $\left.^{\circledast}\right)$ on July $21^{\text {st and }}$ September $3^{\text {rd }}$. Tobradex ${ }^{\oplus}$ ointment was discontinued but prednisolone drops continued four to six times daily. When no significant improvement was noted, further diagnostic evaluation included fluorescein angiogram, vitreous biopsy and rheumatologic evaluation for consideration of Tubulointerstitial Nephritis and Uveitis (TINU) or other autoimmune or rheumatologic conditions because of ongoing abnormal urinary findings.

After six weeks of intensive corticosteroid ophthalmic therapy, the patient was noted to have developed stigmata of adrenal suppression including weight gain; rapid development of striae over trunk, back, legs and arms; fat deposition in both cheeks and upper back; profound hirsuitism on her face, chest and back; and mild elevation in blood pressure (BP 140/88). Both an abnormal random cortisol and fasting cortisol stimulation test confirmed the diagnosis of adrenal suppression. Nasolacrimal plugs were placed to attempt to decrease the systemic absorption of the topically administered corticosteroids and decrease the number of doses per day required for treatment of her uveitis. The patient's antiretroviral medications were discontinued while ophthalmic corticosteroid therapy needed to be intensified. Three weeks after stopping antiretroviral agents, repeat fasting cortisol stimulation test was significantly improved and there was a gradual improvement in the physical stigmata associated with adrenal suppression. However, during the interruption of antiretroviral treatment the patient's CD4 count dropped precipitously and she developed thrombocytopenia with a platelet nadir of $<10,000 / \mu \mathrm{L}$. From October until February, monthly infusions of high dose intravenous immunoglobulin (IVIG) were administered for treatment of thrombocytopenia as well as

*Corresponding author: Diana F Clarke, Boston Medical Center, Section of Pediatric Infectious Diseases, Boston, Massachusetts 02118, United States, Boston, Massachusetts 02118, USA, Tel: 017817711196; Fax: 016174147230; E-mail: diana.clarke@bmc.org

Received June 30, 2014; Accepted August 03, 2014; Published August 13, 2014

Citation: Clarke DF, Moloney C, Cooper ER (2014) latrogenic Cushing's Syndrome Due to Pharmacokinetic Interaction of Intra-Ocular Corticosteroids and Lopinavir/ Ritonavir in an HIV-Infected Adolescent: A Case Report. J AIDS Clin Res 5: 339. doi:10.4172/2155-6113.1000339

Copyright: (C) 2014 Clarke DF, et al. This is an open-access article distributed under the terms of the Creative Commons Attribution License, which permits unrestricted use, distribution, and reproduction in any medium, provided the original author and source are credited. 
ophthalmic findings. Because of concern that unsuppressed HIV viral replication was contributing to thrombocytopenia, antiretroviral agents were restarted in December. A new regimen of antiretroviral agents was initiated which excluded a protease inhibitor so as to avoid the potential for drug interactions with ophthalmic corticosteroids. Previous failure on a Non-Nucleoside Reverse Transcriptase-based (NNRTI) regimen that included nevirapine, the presence of multiple HIV resistance mutations, and her young age limited the options for therapy. The patient was begun on a protease inhibitor-sparing HAART regimen of raltegravir, maraviroc, and abacavir+zidovudine+lamivudine (Trizivir ${ }^{\circledR}$ ) and demonstrated a good virologic and immunologic response over the following weeks.

The patient's ophthalmic findings however continued to worsen, with loss of visual acuity, and the development of an epi-retinal membrane and cataract thought possibly to be complications of the vitreous biopsy. At the same time, there was a worsening of renal findings, with more active sediment and red cell casts noted for the first time in the urinalysis. After much discussion concerning the advisability of performing a renal biopsy in a child with a solitary kidney, the biopsy was performed and a diagnosis of a lupus-like nephritis was made. Despite concern of producing further immune suppression in a patient with HIV infection, treatment with mycophenolatemofetil (Cellcept $\left.{ }^{\oplus}\right)$ was started. There was gradual but steady improvement in her renal, ophthalmic, and hematologic conditions. . The patient's current HIV viral load is undetectable and her CD4 is stable at $568 / \mathrm{mm}^{3}$ and $26 \%$. She continues on antiretroviral regimen consisting of maraviroc, raltegravir and Trizivir; and every other day prednisolone acetate ophthalmic suspension.

\section{Discussion}

We believe this represents the first case of iatrogenic Cushing's syndrome with severe adrenal suppression involving intensive ocular administration of corticosteroids in an adolescent receiving lopinavir/ ritonavir therapy. Limited studies of ocular administration of intensive topical corticosteroids suggest the potential for adrenal suppression. Systemic absorption of topically administered corticosteroids may occur via the ocular or nasal mucous membranes directly into the systemic circulation. In volunteers administered hourly dexamethasone $0.1 \%$ drops for 6 days, a $50 \%$ decrease was observed in endogenous cortisol production [1]. Post-operative cataract patients receiving hourly dexamethasone $0.1 \%$ drops for 3 days had a statistically significant decrease in plasma cortisol levels [2].

Several case reports of iatrogenic Cushing's syndrome in children and adults receiving intensive topical corticosteroids suggest that ocular steroids may carry a similar risk of systemic toxicity as those seen associated with other routes of administration [3-6]. Treatment of uveitis generally includes topical glucocorticoids such as prednisolone $1 \%$ and use of dexamethasone-containing ointments at night. Dosing frequency depends on the intensity of inflammation. Posterior uveitis or panuveitis generally do not respond well to topical therapy alone and periocular injections either by subtenon or intravitreal administration of a long-acting corticosteroid such as Triamcinolone Acetonide (TA) may be required. Significant anti-inflammatory effects may continue for weeks to months after periocular administration. Pharmacokinetic studies have demonstrated that vitreous concentrations of triamcinolone acetonide persist for weeks to months either after subtenon or intravitreal administration [7-9]. Limited data confirm that intravitreal administration of TA can produce detectable serum concentrations [10]. In addition, underlying abnormalities in adrenal function often found in HIV infected patients may predispose those receiving corticosteroids and ritonavir-containing regimens to significant drugdrug interactions.

Ritonavir is a potent cytochrome p450 CYP3A4 inhibitor. It is frequently used as part of protease inhibitor based antiretroviral therapy acting as a pharmacokinetic enhancer. Ritonavir acts by inhibiting the metabolism of the other protease inhibitor, therefore increasing plasma concentrations of the second protease inhibitor. This allows for more consistent plasma concentrations of the protease inhibitor ensuring improved efficacy and higher trough concentrations. However, there are multiple drug interactions associated with ritonavir use, and it is important to carefully review all co-administered medications in patients receiving treatment for HIV infection or any associated condition.

Most administered corticosteroids are metabolized via CYP3A4 metabolic pathway. Even short-term corticosteroid therapy has been associated with iatrogenic Cushing's syndrome and adrenal suppression in HIV-infected patients receiving protease inhibitors. Pharmacokinetic drug interactions caused by inhibition of the metabolism by ritonavir of various corticosteroids have been demonstrated with oral, inhaled (including intranasal), and intra-articular administration [11-16].

There have been multiple case reports in both adults and children who have developed iatrogenic Cushing's syndrome or adrenal failure while receiving fluticasone by oral inhalation or intranasal administration and ritonavir for treatment of asthma or allergic rhinitis [12]. Co-administration of inhaled fluticasone with ritonavir is not recommended unless the potential benefit outweighs the risk of systemic corticosteroid side effects. When discontinuing inhaled corticosteroids, a gradual taper is recommended to avoid corticosteroid withdrawal symptoms. Patients who develop Cushing's syndrome may require replacement therapy with corticosteroids and gradual tapering with special consideration of the potential use of "stress dose" replacement steroids in times of acute illness or trauma. Iatrogenic Cushing's syndrome and secondary adrenal suppression have recently been reported in adult patients receiving ritonavir with a single intraarticular or epidural injection of triamcinolone acetonide [13-16]. Duration of hypothalamic pituitary axis suppression was from 5-8 months in these case reports, suggesting the persistence of systemic triamcinolone plasma concentrations and prolonged suppression of adrenal function.

Although there are limited data, drug interactions with periocular corticosteroids and ritonavir-containing regimens can potentially occur. Because we did not measure prednisolone, dexamethasone, or triamcinolone plasma concentrations in our patient, we do not know which of the agents was responsible for the drug-drug interaction, or whether it was the combination of all three exogenous corticosteroid ophthalmic preparations.

We conclude that intensive ocular administration of corticosteroids for inflammatory eye diseases have the potential to interact with ritonavir in HIV patients receiving boosted protease inhibitor therapy and these patients should be monitored for signs and symptoms of iatrogenic Cushing's syndrome and adrenal suppression. Alternative antiretroviral therapies should be considered when possible if chronic corticosteroid therapy cannot be avoided.

\section{References}

1. Burch PG, Migeon CJ (1968) Systemic absorption of topical steroids. See comment in PubMed Commons below Arch Ophthalmol 79: 174-176.

2. Roters S, Aspacher F, Diestelhorst M (1996) The influence of dexamethasone $0.1 \%$ eye drops on plasma cortisol and ACTH concentrations after cataract surgery. Ophthalmologica 210: 211-214 
Citation: Clarke DF, Moloney C, Cooper ER (2014) latrogenic Cushing's Syndrome Due to Pharmacokinetic Interaction of Intra-Ocular Corticosteroids and Lopinavir/Ritonavir in an HIV-Infected Adolescent: A Case Report. J AIDS Clin Res 5: 339. doi:10.4172/2155-6113.1000339

3. Gray C (2006) Systemic toxicity with topical ophthalmic medications in children. Paed Perinat Drug Ther 7: 23-29.

4. Messina MF, Valenzise M, Aversa S, Arrigo T, De Luca F (2009) latrogenic Cushing syndrome caused by ocular glucocorticoids in a child. BMJ Case Rep.

5. Afandi B, Toumeh MS, Saadi HF (2003) Cushing's syndrome caused by unsupervised use of ocular glucocorticoids. Endocr Pract 9: 526-529.

6. Chiang MY, Sarkar M, Koppens JM, Milles J, Shah P (2006) Exogenous Cushing's syndrome and topical ocular steroids. Eye (Lond) 20: 725-727.

7. Inoue M, Takeda K, Morita K, Yamada M, Tanigawara Y, et al. (2004) Vitreous concentrations of triamcinolone acetonide in human eyes after intravitreal or subtenon injection. See comment in PubMed Commons below Am J Ophthalmol 138: 1046-1048.

8. Thomas ER, Wang J, Ege E, Madsen R, Hainsworth DP (2006) Intravitrea triamcinolone acetonide concentration after subtenon injection. See comment in PubMed Commons below Am J Ophthalmol 142: 860-861.

9. Beer PM, Bakri SJ, Singh RJ, Liu W, Peters GB, et al. (2003) Intraocular concentration and pharmacokinetics of triamcinolone acetonide after a single intravitreal injection. Ophthalmology 110: 681-686.

10. Degenring RF, Jonas JB (2004) Serum levels of triamcinolone acetonide after intravitreal injection. See comment in PubMed Commons below Am J Ophthalmol 137: 1142-1143.
11. Penzak SR, Formentini E, Alfaro RM, LongM, Natarajan V, et al. (2005) Prednisolone pharmacokinetics in the presence and absence of ritonavir after oral prednisone administration to healthy volunteers. J Acquir Immune Defic Syndr 40: $573-580$

12. Foisy MM, Yakiwchuk EM, Chiu I, Singh AE (2008) Adrenal suppression and Cushing's syndrome secondary to an interaction between ritonavir and fluticasone: a review of the literature. See comment in PubMed Commons below HIV Med 9: 389-396.

13. Dort K, Padia S, Wispelwey B, Moore CC (2009) Adrenal suppression due to an interaction between ritonavir and injected triamcinolone: a case report. See comment in PubMed Commons below AIDS Res Ther 6: 10.

14. Ramanathan R, Pau AK, Busse KH, Zemskova M, Nieman L, et al. (2008) latrogenic Cushing syndrome after epidural triamcinolone injections in an HIV type 1-infected patient receiving therapy with ritonavir-lopinavir. See comment in PubMed Commons below Clin Infect Dis 47: e97-99.

15. Yombi JC, Maiter D, Belkhir L, Nzeusseu A, Vandercam B (2008) latrogenic Cushing's syndrome and secondary adrenal insufficiency after a single intraarticular administration of triamcinolone acetonide in HIV-infected patients treated with ritonavir. Clin Rheumatol 27: S79-S82.

16. Hyle EP, Wood BR, Backman ES, Noubary F, Hwang J, et al. (2013) High frequency of hypothalamic-pituary-adrenal axis dysfunction after local corticosteroid injection in HIV-infected patients on protease inhibitor therapy. J Acquir Immune Defic Syndr 63: 602-608. 